\title{
Adaptive partition intuitionistic fuzzy time series forecasting model
}

\author{
Xiaoshi Fan*, Yingjie Lei, and Yanan Wang \\ Air and Missile Defense College, Air Force Engineering University, Xi' an 710051, China
}

\begin{abstract}
To enhance the accuracy of intuitionistic fuzzy time series forecasting model, this paper analyses the influence of universe of discourse partition and compares with relevant literature. Traditional models usually partition the global universe of discourse, which is not appropriate for all objectives. For example, the universe of the secular trend model is continuously variational. In addition, most forecasting methods rely on prior information, i.e., fuzzy relationship groups (FRG). Numerous relationship groups lead to the explosive growth of relationship library in a linear model and increase the computational complexity. To overcome problems above and ascertain an appropriate order, an intuitionistic fuzzy time series forecasting model based on order decision and adaptive partition algorithm is proposed. By forecasting the vector operator matrix, the proposed model can adjust partitions and intervals adaptively. The proposed model is tested on student enrollments of Alabama dataset, typical seasonal dataset Taiwan Stock Exchange Capitalization Weighted Stock Index (TAIEX) and a secular trend dataset of total retail sales for social consumer goods in China. Experimental results illustrate the validity and applicability of the proposed method for different patterns of dataset.
\end{abstract}

Keywords: intuitionistic fuzzy set, time series, forecasting, vector operator matrix, order deciding, adaptive partition.

DOI: $10.21629 /$ JSEE.2017.03.18

\section{Introduction}

Time series analysis is an availability method for prediction. In reality, conventional methods cannot deal with uncertain information such as linguistic variable. Song et al. proposed a fuzzy time series (FTS) forecasting model by combining the fuzzy set theory with time series analysis $[1,2]$. Chen et al. proposed a simplified arithmetic replaced max-min composition operations, which improves the operation efficiency [3]. Hwang et al. presented a successful FTS prediction model to forecast the enrollments of the University of Alabama [4]. In [5], Huarng et al. proposed

Manuscript received July 13, 2016.

*Corresponding author.

This work was supported by the National Natural Science Foundation of China (61309022). a method by adding a heuristic function to improve the previous model. The application of neural networks and particle swarm optimization (PSO) in FTS prediction were discussed in [6,7], respectively, both models were used to forecast Taiwan Futures Exchange (TAIFEX). Song analyzed the linear FTS by defining fuzzy plus and minus operators, and proposed a forecasting model based on autocorrelation functions [8]. Cheng et al. presented a fuzzy time series forecasting method based on entropy and trapezoid fuzzification [9]. Considering about the influence of multiple attribute on input, a multivariate stochastic fuzzy model [10] and an adaptive expectation forecasting model were proposed [11].

For reforming the forecasting accuracy, several literature researched the high-order fuzzy time series models instead of the first-order model. In [12], Chen constructed a high-order fuzzy time series model, then Own et al. proposed another heuristic high-order method to improve it [13]. Lee et al. considered more factors for prediction and distinguished the main factor from the second factor, then proposed a two-factor high-order FTS model [14]. Then several different high-order FTS forecasting models were presented [15-19]. On the other hand, it is shown that the intervals length of universe of discourse influences the prediction accuracy greatly. Fuzzy clustering is the most popular intervals partition method [17,18,20-23]. PSO [24,25], tabu search [26], information granules [27,28], and ant colony optimization [29] have been applied to seek appropriate intervals. In general, the typical time series patterns can be sorted as secular trend, seasonal variation trend, cyclical variation trend and irregular variation trend [30]. Unlike the stationary of trend time series pattern, Liu et al. proposed an improved forecasting model focusing on forecasting seasonal time series [31], which demonstrated that the universe of discourse partition and relationship generated different performance in different patterns. $\mathrm{Li}$ et al. presented a deterministic forecasting model based on the fuzzy $c$-means (FCM) clustering method to address 
the two-factor forecasting problem [32]. Liu proposed an incorporate clustering method and introduced the $\alpha$ factor method to adjust forecasting output value [11]. In [33], a hierarchical clustering algorithm was presented to deal with non-linear time series. In a word, the order deciding and the universe of discourse partition are two important aspects influencing the forecasting accuracy.

Taking a close examination on existing methods, we can conclude that more accurate partition can lead to better forecasting results. No matter what equal partition or clustering algorithm was adopted, these models need to discuss the whole universe of discourse [3,4,11,12,16,18], and the computational complexity will grow along with the scale of data. Usually, these methods are applied to small scale, stable range or seasonal variation model, such as enrollments and temperature of weather data, which illuminates that the applicability and generality of these models are limited. As an extension of fuzzy sets, the intuitionistic fuzzy sets theory proposed by Atanassov can depict the fuzzy object more clearly [34]. In [35], Castillo et al. introduced the intuitionistic fuzzy reasoning to time series analysis. After that, some intuitionistic fuzzy time series (IFTS) methods were successively proposed [36,37]. Zheng et al. constructed a kind of IFTS models [38] to handle long-term forecasting problem [39]. Above researches indicate that the fusion of intuitionistic fuzzy sets and time series prediction is a new development trend. To address the difficulty in optimal order number determination and the limitation of partition in existent models, in this paper, we attempt to propose a novel IFTS forecasting model, with the purpose of increasing the forecasting accuracy and improving the model adaptability. The proposed model offers an intuitionistic fuzzification method of historical data and the appropriate order number determination algorithms. Meanwhile the proposed IFTS model based on adaptive partition is able to deal with conventional FTS problems and overcome the limitation of the universe of discourse. Our model will enhance the generalization ability of FTS forecasting.

The rest of this paper is organized as follows. In Section 2, some definitions of intuitionistic FTS and related work are given. Then, the order deciding and adaptive partition algorithms are presented particularly. In Section 3, an integrated adaptive partition IFTS forecasting model is constructed and compared with other methods by being applied to enrollments of Alabama. In Section 4, the proposed model is implemented on two different dataset patterns, seasonal pattern, TAIEX and secular trend pattern, the monthly total retail sales of social consumer goods (TRSSCG). Finally, some conclusions are drawn in Section 5 .

\section{IFTS and algorithms}

\subsection{IFTS definitions}

In $[1,2]$, the FTS and basic theory have been proposed. Here, some definitions are introduced briefly.

Definition 1 Assume $A$ is a fuzzy set defined in universe of discourse $U$, which is represented as

$$
A=f\left(\mu_{1}\right) / A_{1}+f\left(\mu_{2}\right) / A_{2}+\cdots+f\left(\mu_{n}\right) / A_{n}
$$

where $f\left(\mu_{i}\right)$ is the membership function of $A, f\left(\mu_{i}\right) \rightarrow$ $[0,1](1 \leqslant i \leqslant n)$ is the connection sign.

Definition 2 Let $\{Y(t), t=0,1, \ldots, n\}$, a subset of $\mathbf{R}$, be the universe of discourse, on which the fuzzy set $f_{i}(t)\left(i=1,2,3, \ldots, n ; f_{i}(t) \in[0,1]\right)$ is defined and $F(t)$ is a collection of $f_{1}(t), f_{2}(t), \ldots, f_{n}(t)$, where $F(t)$ can be denoted as the linguistic variable, then $F(t)$ is called a fuzzy time series defined on $Y(t)$.

Definition 3 Assume that an FTS $F(t)$ is decided by $F(t-1)$ only, then $F(t)=F(t-1) \circ \boldsymbol{R}(t, t-1)$, is a fuzzy synthetic operator, which is called a first-order model of $F(t)$, where $\boldsymbol{R}(t, t-1)$ is the fuzzy relationship matrix. If for any $t, \boldsymbol{R}(t, t-1)=\boldsymbol{R}(t-1, t-2)$, $F(t)=F(t-1) \circ \boldsymbol{R}$ is called a time-invariant fuzzy series, otherwise it is a time-variant fuzzy series.

Definition 4 Assume that an FTS $F(t)$ is decided by $F(t-1), F(t-2), \ldots, F(t-m)$,

$$
\begin{gathered}
F(t)=F(t-1) \times F(t-2) \times \cdots \times \\
F(t-m) \circ \boldsymbol{R}(t, t-m)
\end{gathered}
$$

where $F(t)$ is called an $m$-order fuzzy time series model, $\boldsymbol{R}(t, t-m)$ is an $m$-order composition fuzzy relationship, $\times$ is the Cartesian product.

Similarly, based on the theories of FTS, some definitions of intuitionistic fuzzy time series are presented particularly as follows:

Definition 5 Let $\{Y(t), t=0,1, \ldots, n\}$ be a time series on universe of discourse $U$, and $A$ be a division set of $U$, namely $\left\{A_{i}, i=1,2, \ldots, k ; \bigcup_{i=1}^{k} A_{i}=U\right\}$, where $A_{i}$ is the language variable. If a time series $F(t)$ composed by $\left\{A_{i}\right\}$ has a pair of membership and non-membership function $\mu_{i}(Y(t)), \gamma_{i}(Y(t)), F_{I}(t)$ is marked as an intuitionistic fuzzy time series on $Y(t)$ and expressed by the following expression.

$$
\begin{gathered}
F_{I}(t)=<\mu_{1}\left(Y(t), \gamma_{1}(Y(t))>/ A_{1}+\right. \\
<\mu_{2}\left(Y(t), \gamma_{2}(Y(t))>/ A_{2}+\cdots+\right. \\
<\mu_{n}\left(Y(t), \gamma_{n}(Y(t))>/ A_{n}\right.
\end{gathered}
$$


where $f_{i}(t)=<\mu_{i}(Y(t)), \gamma_{i}(Y(t))>/ L_{i}$ denotes the membership and non-membership functions of IFS $Y(t)$ with respect to $A_{i}$.

Definition 6 Assume that an IFTS $F_{I}(t)$ is merely decided by $F_{I}(t-1)$, then $F_{I}(t)=F_{I}(t-1) \circ \boldsymbol{R}_{I}(t, t-1)$ is called a first-order IFTS, where $\boldsymbol{R}_{I}(t, t-1)$ is the intuitionistic fuzzy relationship (IFR) matrix with $\boldsymbol{R}_{i j}=<$ $\boldsymbol{R}\left(\mu_{i j}\right), \boldsymbol{R}\left(\gamma_{i j}\right)>=F_{I}^{\mathrm{T}}(t) \circ F_{I}(t-1)=\left[R_{i j}\right]_{r \times r}$. If the membership and non-membership functions of $F_{I}(t)$ and $F_{I}(t-1)$ are $<\mu_{1 i}, \gamma_{1 i}>$ and $<\mu_{2 i}, \gamma_{2 i}>$, respectively, $\boldsymbol{R}\left(\mu_{i j}\right)$ and $\boldsymbol{R}\left(\gamma_{i j}\right)$ can be calculated by the following expression.

$$
\begin{aligned}
\boldsymbol{R}\left(\mu_{i j}\right) & = \\
\bigvee_{k=1}^{r}\left(\mu_{1 i k} \wedge \mu_{2 i k}\right), \boldsymbol{R}\left(\gamma_{i j}\right) & =\bigwedge_{k=1}^{r}\left(\gamma_{1 i k} \vee \gamma_{2 i k}\right) .
\end{aligned}
$$

For any $t$, if $\boldsymbol{R}_{I}(t, t-1)=\boldsymbol{R}_{I}(t-1, t-2)$, then $F_{I}(t)=F_{I}(t-1) \circ \boldsymbol{R}_{I}$ is called a time-invariant intuitionistic fuzzy series; otherwise, it is called a time-variant intuitionistic fuzzy series.

Definition 7 Assume that an IFTS $F_{I}(t)$ is decided by $F_{I}(t-1), F_{I}(t-2), \ldots, F_{I}(t-m)$, we can get a high-order intuitionistic fuzzy time series relationship expression as follows:

$$
\begin{gathered}
F_{I}(t)=F_{I}(t-1) \times F_{I}(t-2) \times \cdots \times \\
F_{I}(t-m) \circ \boldsymbol{R}_{I}(t, t-m)
\end{gathered}
$$

where $\boldsymbol{R}(t, t-m)$ is an $m$-order composition fuzzy relationship. Furthermore, the intuitionistic fuzzy relationship can be obtained as

$$
\begin{gathered}
\boldsymbol{R}_{I}(t, t-m)=f_{I}(t) \times f_{I}(t-1) \cup f_{I}(t-1) \times f_{I}(t-2) \cup \\
\cdots \cup f_{I}(t-m+1) \times f_{I}(t-m)
\end{gathered}
$$

In [1 -5], some FTS forecasting models have been constructed. In general, an FTS forecasting model includes several aspects: (i) the universe of discourse partition; (ii) historical time series data fuzzification; (iii) established fuzzy relationship; (iv) forecasting and defuzzification. According to prototype, a novel adaptive IFTS forecasting model is presented in this paper and the framework is shown in Fig. 1. The proposed model includes two parts, data training and data forecasting. The number $m$ is decided by the order deciding algorithm and the universe of discourse is divided by the adaptive partition algorithm.
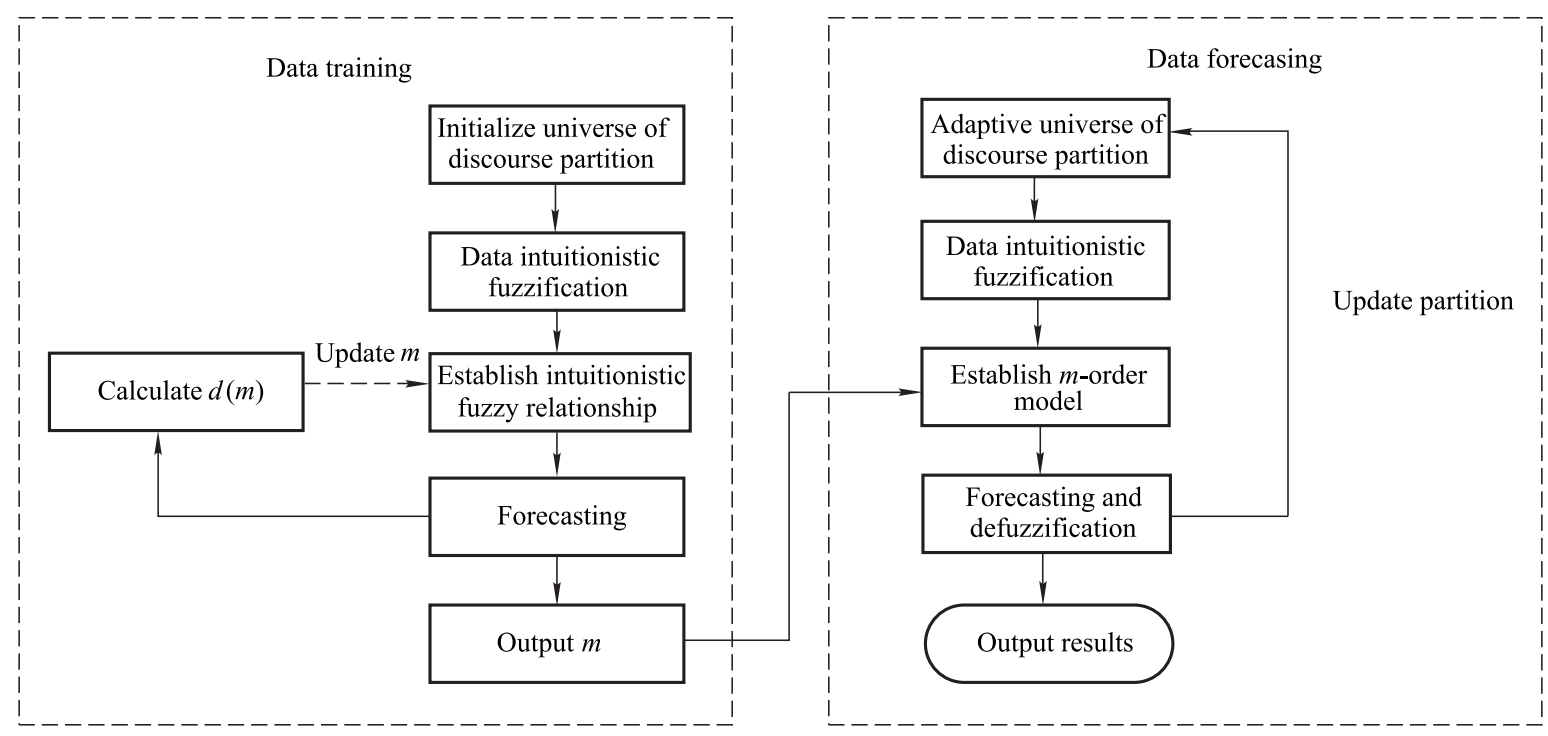

Fig. 1 Framework of the adaptive partition IFTS model

\subsection{Order deciding algorithm}

In the data training part, the $m$-order deciding algorithm is used to decide an appropriate order. Detailed procedure is presented stepwise as follows and the flow chart is presented in Fig. 2.

Step 1 To compare with other methods clearly, we use equal intervals as the initial partition. Define the universe of discourse $U=\left[D_{\min }-D_{1}, D_{\max }-D_{2}\right]$, where $D_{\min }$ and $D_{\max }$ are minimum and maximum of historical data. In order to highlight fuzzy attribute of $U$, which is divided into $n$ overlap parts, $U=u_{1} \cup u_{2} \cup \cdots \cup u_{n}$, the midpoint of current interval is regarded as the start point of next interval.

Step 2 Let $A_{i}$ represent the linguistic variable, then, $A_{i}=\sum_{j=1}^{n}<\mu_{i j}, \gamma_{i j}>/ u_{j}$, where $\mu_{i j}$ and $\gamma_{i j}$ are mem- 
bership and non-membership of $A_{i}$ belong to $u_{i}$. The $\mu_{i j}$ and $\gamma_{i j}$ can be calculated as

$$
\begin{gathered}
<\mu_{i j}, \gamma_{i j}>= \\
\left\{\begin{array}{c}
<1-\left|\frac{2 d_{i}-(A+B)}{\lambda(B-A)}\right|,\left|\frac{2 d_{i}-(A+B)}{B-A}\right|>, \\
d_{i} \in u_{j} \\
<0,1>, \text { otherwise }
\end{array}\right.
\end{gathered}
$$

where $A$ and $B$ are boundaries of interval, $d_{i}$ is the historical data, $\lambda$ is an adjustment factor of hesitancy degree.

Step 3 The linguistic fuzzification result based on interval which the data fall into, and then, intuitionistic fuzzy logical relationships (IFLR) are obtained. We can get a relationship $A_{i}, A_{j} \rightarrow A_{i+1}, A_{j+1}$, here, a pair of results $A_{i}$ and $A_{j}$ is called the current state of intuitionistic fuzzy logical relationship and $A_{i+1}$ and $A_{j+1}$ is called the next state of intuitionistic fuzzy logical relationship.

Step $4 m$ (initial $m=1$ ) intuitionistic fuzzy relationship pairs are obtained, namely, $A_{t-m} \rightarrow$ $A_{t-m+1}, A_{t-m+1} \rightarrow A_{t-m+2}, \ldots, A_{t-1} \rightarrow A_{t}$. According to (4), we can calculate $\boldsymbol{R}_{k}=<\boldsymbol{R}\left(\mu_{i j}\right), \boldsymbol{R}\left(\gamma_{i j}\right)>$ $(k=1,2, \ldots, m)$, respectively. Finally, the intuitionistic fuzzy relationship is calculated by

$$
\boldsymbol{R}_{I}=<\bigcup_{k=1}^{m} \boldsymbol{R}_{k}\left(\mu_{i j}\right), \bigcap_{k=1}^{m} \boldsymbol{R}_{k}\left(\gamma_{i j}\right)>\text {. }
$$

Step 5 These IFLRs are gathered to groups. If there is one current state corresponding to several next states, the IFLRs are regarded as one group. When forecasting, the next state is decided by the mean current relationship in intuitionistic fuzzy logical relationship groups (IFLRG). For example, if the current intuitionistic fuzzy logical relationship group is $<A_{4} A_{5} \rightarrow A_{3} A_{4}, A_{4} A_{5} \rightarrow A_{4} A_{5}$, $A_{4} A_{5} \rightarrow A_{5} A_{6}>$, the next state is forecasted as $A_{4} A_{5}$.

Step 6 Forecasted output $F_{I}(t+1)$ is calculated based on (5). The nonzero $<\mu_{i}, \gamma_{i}>/ u_{i}$ belonging to the relevant intuitionistic fuzzy relationship is chosen according to the triangular membership function and the precise output value is calculated by the following formula.

$$
\begin{gathered}
\operatorname{def}(F(t+1))= \\
\frac{1}{n} \sum_{i=1}^{n}\left(B_{i}-\frac{B_{i}-A_{i}}{4}\left(1+\mu_{i}-\gamma_{i}\right)\right) .
\end{gathered}
$$

Based on the defuzzification method, the outputs rely on nearby two intervals, here $n=2$.

Step 7 The intuitionistic fuzzy distance square error (IFDSE) $d(\mu(m), \gamma(m))$ is calculated by (10).

$$
d(\mu(m), \gamma(m))=
$$

$$
\frac{1}{l} \sum_{i=1}^{l} \sum_{j=1}^{n}\left[\left(\mu_{i j}-\mu_{i j}^{\prime}\right)^{2}+\left(\gamma_{i j}-\gamma_{i j}^{\prime}\right)^{2}\right]
$$

where $\mu_{i j}$ and $\gamma_{i j}$ are calculated by (7), $\mu_{i j}^{\prime}$ and $\gamma_{i j}^{\prime}$ are calculated by (5). The former pair of membership and nonmembership functions is actual values and the later pair is forecasted values. $l$ is the capacity of training data. Update $m=m+1$, according to (11), we choose $m$ from the minimum of intuitionistic fuzzy mean distance square error or the variety between $d(m)$ and $d(m+1)$ which is small enough, where $\varepsilon$ is permissible error.

$$
\left\{\begin{array}{l}
d(\mu(m-1), \gamma(m-1)) \leqslant d(\mu(m), \gamma(m)) \leqslant \\
d(\mu(m+1), \gamma(m+1)) \\
\quad \text { or } \\
d(\mu(m), \gamma(m))-d(\mu(m+1), \gamma(m+1)) \leqslant \varepsilon
\end{array} .\right.
$$

Step 8 Output an appropriate value $m$.

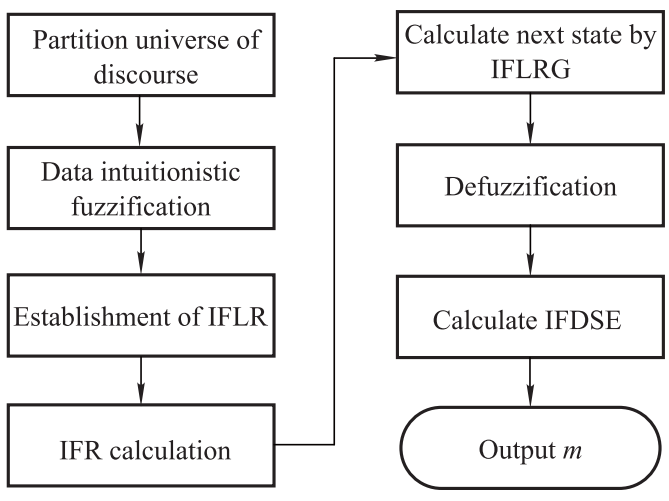

Fig. 2 Flow of the $m$-order deciding algorithm

\subsection{Adaptive partition algorithm}

During the procedure of IFTS model construction, the establishment of fuzzy relationship is crucial to the accuracy of forecasting. In [1,2], the current fuzzy relationship was synthesized by previous $m$ relationships and regarded as next relationship for prediction. The virtue of this method is convenient, but the precision of forecasting is not high. In $[3,11,32,36]$, the presented methods improved the accuracy of forecasting by the fuzzy relationship obtained from fuzzy relationship groups. However, such relationship groups are prior information, then the forecasting error will be very large when the next state does not appear in groups before. The applicability of these methods is limited. Furthermore, type of dataset impacts universe of discourse partition. For example, the global universe of a linear time series dataset is divided into parts that may cause explosive growth of fuzzy relationship library. The accuracy is positively correlated to the computational complexity. In this situation, constructing relationship groups are not appropriate. 
To overcome these shortages, we propose a new method to calculate the intuitionistic fuzzy relationship in a forecasting model based on vector operator matrix $\boldsymbol{O}(t)$.

Definition 8 Assume that an IFTS $F_{I}(t)$ is decided by $F_{I}(t-1), F_{I}(t-2), \ldots, F_{I}(t-m), \boldsymbol{R}_{I}(t, t-m)$ is calculated based on (4) and $m$ intuitionistic fuzzy logic relationships $A_{i} \rightarrow A_{j}$ are available, then we have

$$
\begin{array}{r}
F_{I}(t+1)=F_{I}(t) \times R_{I}(t-m) \times \boldsymbol{O}(t) \\
\boldsymbol{O}(t)=\left\{\begin{array}{l}
\boldsymbol{D}^{r}, d>0 \\
\boldsymbol{E}, \quad d=0 \\
\boldsymbol{D}^{\mathrm{T}(-r)}, \quad d<0
\end{array}\right. \\
\boldsymbol{D}=\left[a_{k, k+1}=1\right]_{p \times p}, \quad k=1,2, \ldots, p-1 \\
r=\left[\frac{1}{m} \sum_{t=1}^{m} i-j\right]
\end{array}
$$

where $\boldsymbol{D}$ is a direction vector matrix, $\boldsymbol{E}$ is the unit matrix, $r$ is the forecasting index and $p$ is the partition value.

For example, assume that there is a 3-order IFTS forecasting model, of which the partition value is five and the fuzzy relationships $<A_{1} \rightarrow A_{3}, A_{3} \rightarrow A_{3}, A_{3} \rightarrow A_{4}>$ are available, then, we can calculate $r=1$,

$$
\boldsymbol{O}(t)=\boldsymbol{D}^{1}=\left[\begin{array}{lllll}
0 & 1 & 0 & 0 & 0 \\
0 & 0 & 1 & 0 & 0 \\
0 & 0 & 0 & 1 & 0 \\
0 & 0 & 0 & 0 & 1 \\
0 & 0 & 0 & 0 & 0
\end{array}\right]
$$

For illustration, assume $A_{4}=0 / u_{1}+0 / u_{2}+0.5 / u_{3}+$ $1 / u_{4}+0 / u_{5}, R_{I}(t-m)=\boldsymbol{E}$, finally, $F_{I}(t+1)=A_{5}=$ $0 / u_{1}+0 / u_{2}+0 / u_{3}+0.5 / u_{4}+1 / u_{5}$ is forecasted based on (5).

In this section, the forecasting part is constructed and the adaptive partition algorithm is shown as follows, and the flow chart is presented in Fig. 3.

Step 1 Set the initial partition $p$. The initial universe of discourse $U$ is divided into $p$ parts with equal interval $\Delta A$, namely, $U=A_{1} \cup A_{2} \cup \cdots \cup A_{p}$, then the midpoint of current interval is set as the start point of next interval.

Step 2 Calculate the linguistic fuzzification results by the interval which data drop into. $\mu_{i j}$ and $\gamma_{i j}$ can be calculated by the same Step 2 of the order deciding algorithm.

Step 3 Get the relationships $A_{i}, A_{j} \rightarrow A_{i+1}, A_{j+1}$ by historical time series data, calculate $r$ and $\boldsymbol{O}(t)$ according to (15) and (13), respectively. The relationship matrix $\boldsymbol{R}_{\mu}$ and $\boldsymbol{R}_{\gamma}$ are calculated by (6).

Step 4 The IFTS forecasting result $F(t+1)$ is calculated based on (12). Forecast fuzzy output $A_{i}, A_{j} \rightarrow A_{i+r}$, $A_{j+r}$ and identify the actual result by the fuzzification. The defuzzification value is calculated based on (9). Here, choose the membership and non-membership functions of $<A_{i+r}, A_{j+r}>$ and $n=2$.

Step 5 Update partition $\Delta A$ and limit by trend of forecasting result. When $r>0$, increase the upper limit and lower limit of universe of discourse, in contrast, when $r<0$, decrease the upper limit and lower limit simultaneously. Keep it unchanged when $r=0$. If the fuzzification results belong to the first or the last interval, we should set $p=p+1$, which indicates that the universe of discourse is not larger enough. If the scale of universe changes drastically, set $\Delta A=2^{n} \Delta A$ or $\Delta A=(1 / 2)^{n} \Delta A$, here, $n=1,2, \ldots$..

Step 6 Jump to Step 4 and calculate the rest fuzzification and defuzzification values.

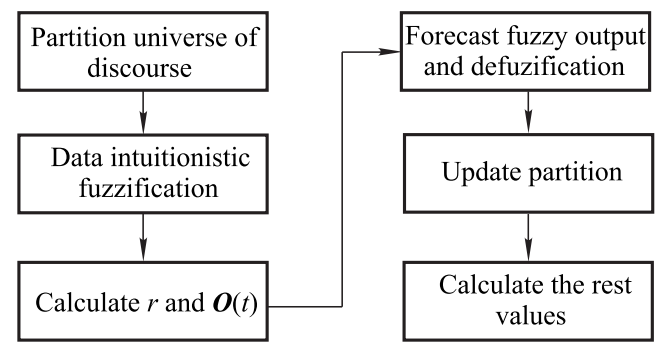

Fig. 3 Flow of the adaptive partition algorithm

There are two cases of defuzzification. Taking membership degree function as an example, in Case 1, the fuzzy output $F$ only locates in the first or the last interval, such as $F_{1}$ and $F_{n}$ in Fig. 4 , and we utilize one membership defuzzification. In Case 2, the fuzzy output $F_{2}$ locates in overlap intervals, and we utilize the mean of two precise values calculated by $\left\langle\mu_{i}, \gamma_{i}>\right.$ respectively as the finial output value.

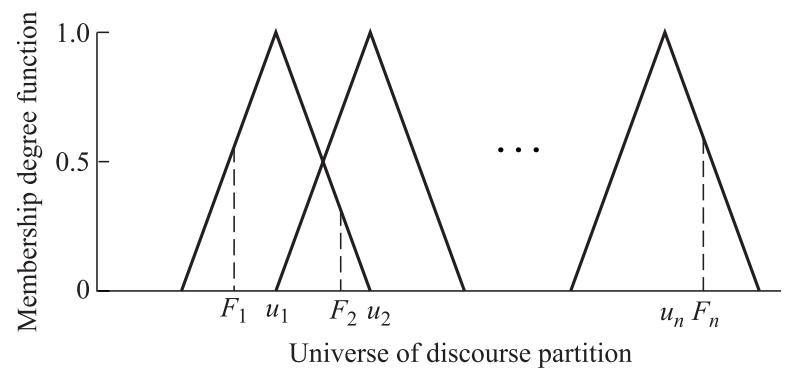

Fig. 4 Cases of defuzzification

\section{Adaptive partition IFTS forecasting model}

In this section, the adaptive partition IFTS forecasting model is presented by implementing on widely used data, student enrollments of Alabama from 1971 to 1992 particular. The effect of the $m$-order deciding and adaptive partition algorithm is validated. 


\subsection{Data training}

The enrollments from 1971 to 1992 are shown in Table 1. In data training part, we utilize the former 12 data as the training specimen to decide number $m$, others as testing data for testifying validity of the order deciding algorithm.

Table 1 Specimen of enrollments

\begin{tabular}{ccc||rcc}
\hline Year & Value & Fuzzification & Year & Value & Fuzzification \\
\cline { 5 - 6 } 1971 & 13055 & $A_{1}, /$ & 1982 & 15433 & $A_{4}, A_{5}$ \\
1972 & 13563 & $A_{1}, A_{2}$ & 1983 & 15497 & $A_{4}, A_{5}$ \\
1973 & 13867 & $A_{1}, A_{2}$ & 1984 & 15145 & $A_{4}, A_{5}$ \\
1974 & 14696 & $A_{3}, A_{4}$ & 1985 & 15163 & $A_{4}, A_{5}$ \\
1975 & 15460 & $A_{4}, A_{5}$ & 1986 & 15984 & $A_{5}, A_{6}$ \\
1976 & 15311 & $A_{3}, A_{4}$ & 1987 & 16859 & $A_{7}, A_{8}$ \\
1977 & 15603 & $A_{5}, A_{6}$ & 1988 & 18150 & $A_{10}, A_{11}$ \\
1978 & 15861 & $A_{5}, A_{6}$ & 1989 & 18970 & $A_{11}, A_{12}$ \\
1979 & 16807 & $A_{7}, A_{8}$ & 1990 & 19328 & $A_{12}, A_{13}$ \\
1980 & 16919 & $A_{7}, A_{8}$ & 1991 & 19337 & $A_{12}, A_{13}$ \\
1981 & 16383 & $A_{6}, A_{7}$ & 1992 & 18876 & $A_{11}, A_{12}$ \\
\hline
\end{tabular}

Firstly, define the universe of discourse $U=[13000$, $20000]$ and partition it as follows: $A_{1}=[13000,14000]$, $A_{2}=\left[\begin{array}{lll}13 & 500,14 & 500\end{array}\right], A_{3}=\left[\begin{array}{lll}14 & 000,15 & 000\end{array}\right]$, $A_{4}=\left[\begin{array}{lll}14 & 500,15 & 500\end{array}\right], A_{5}=\left[\begin{array}{lll}15 & 000,16 & 000\end{array}\right]$, $A_{6}=\left[15500,16\right.$ 500], $A_{7}=\left[\begin{array}{ll}16 & 000,17000\end{array}\right], A_{8}=$ [16 500,17 500], $A_{9}=\left[\begin{array}{lll}17 & 000,18 & 000\end{array}\right], A_{10}=$ [17 500, 18 500], $A_{11}=\left[\begin{array}{lll}18 & 000,19 & 000\end{array}\right], A_{12}=$ [18 500, 19 500], $A_{13}=$ [19 000, 20 000]. The linguistic fuzzification result based on interval which enrollments fall into and intuitionistic fuzzy logical relationships are obtained shown in Table 1.

Between 1973 and 1974, we can get a relationship $A_{1}$, $A_{2} \rightarrow A_{3}, A_{4}$, and cluster these fuzzy logical relationships to groups, if there is one current state corresponding to several next states, regard them as one group. For example, the current intuitionistic fuzzy logical relationship group is $<A_{4} A_{5} \rightarrow A_{3} A_{4}, A_{4} A_{5} \rightarrow A_{4} A_{5}, A_{4} A_{5} \rightarrow A_{5} A_{6}>$, and the next state is forecasted $A_{4} A_{5}$. The logical relationships of enrollments and the logical relationship groups are shown in Table 2 and Table 3.

Table 2 Intuitionistic fuzzy logical relationships of enrollments

\begin{tabular}{cc}
\hline \multicolumn{2}{c}{ Relationship groups } \\
\hline$A_{1} \rightarrow A_{1}, A_{2}$ & $A_{1}, A_{2} \rightarrow A_{1}, A_{2}$ \\
$A_{1}, A_{2} \rightarrow A_{3}, A_{4}$ & $A_{3}, A_{4} \rightarrow A_{4}, A_{5}$ \\
$A_{4}, A_{5} \rightarrow A_{3}, A_{4}$ & $A_{3}, A_{4} \rightarrow A_{5}, A_{6}$ \\
$A_{5}, A_{6} \rightarrow A_{5}, A_{6}$ & $A_{5}, A_{6} \rightarrow A_{7}, A_{8}$ \\
$A_{7}, A_{8} \rightarrow A_{7}, A_{8}$ & $A_{7}, A_{8} \rightarrow A_{6}, A_{7}$ \\
$A_{6}, A_{7} \rightarrow A_{4}, A_{5}$ & $A_{4}, A_{5} \rightarrow A_{4}, A_{5}$ \\
$A_{4}, A_{5} \rightarrow A_{4}, A_{5}$ & $A_{4}, A_{5} \rightarrow A_{4}, A_{5}$ \\
$A_{4}, A_{5} \rightarrow A_{4}, A_{5}$ & $A_{4}, A_{5} \rightarrow A_{5}, A_{6}$ \\
$A_{5}, A_{6} \rightarrow A_{7}, A_{8}$ & $A_{7}, A_{8} \rightarrow A_{10}, A_{11}$ \\
$A_{10}, A_{11} \rightarrow A_{11}, A_{12}$ & $A_{11}, A_{12} \rightarrow A_{12}, A_{13}$ \\
$A_{12}, A_{13} \rightarrow A_{11}, A_{12}$ & \\
\hline
\end{tabular}

Table 3 Intuitionistic fuzzy logical relationship groups

\begin{tabular}{ccc}
\hline \multicolumn{3}{c}{ Relationship groups } \\
\hline$A_{1} \rightarrow A_{1}, A_{2}$ & & \\
$A_{1}, A_{2} \rightarrow A_{1}, A_{2}$ & $A_{1}, A_{2} \rightarrow A_{3}, A_{4}$ & \\
$A_{3}, A_{4} \rightarrow A_{4}, A_{5}$ & $A_{3}, A_{4} \rightarrow A_{5}, A_{6}$ & \\
$A_{4}, A_{5} \rightarrow A_{3}, A_{4}$ & $A_{4}, A_{5} \rightarrow A_{4}, A_{5}$ & $A_{4}, A_{5} \rightarrow A_{5}, A_{6}$ \\
$A_{5}, A_{6} \rightarrow A_{5}, A_{6}$ & $A_{5}, A_{6} \rightarrow A_{7}, A_{8}$ & \\
$A_{6}, A_{7} \rightarrow A_{4}, A_{5}$ & & \\
$A_{7}, A_{8} \rightarrow A_{7}, A_{8}$ & $A_{7}, A_{8} \rightarrow A_{6}, A_{7}$ & $A_{7}, A_{8} \rightarrow A_{10}, A_{11}$ \\
$A_{10}, A_{11} \rightarrow A_{11}, A_{12}$ & & \\
$A_{11}, A_{12} \rightarrow A_{12}, A_{13}$ & \\
$A_{12}, A_{13} \rightarrow A_{11}, A_{12}$ & \\
\hline
\end{tabular}

Set $m=1$, the IFDSE $d(\mu(m), \gamma(m))$ can be calculated by (10), update $m=m+1$ and the results are shown in Fig. 5. Then the mean square error $d(m)$ are calculated as follows: $d(1)=0.4475, d(2)=0.2976, d(3)=0.2477$, $d(4)=0.3570$.

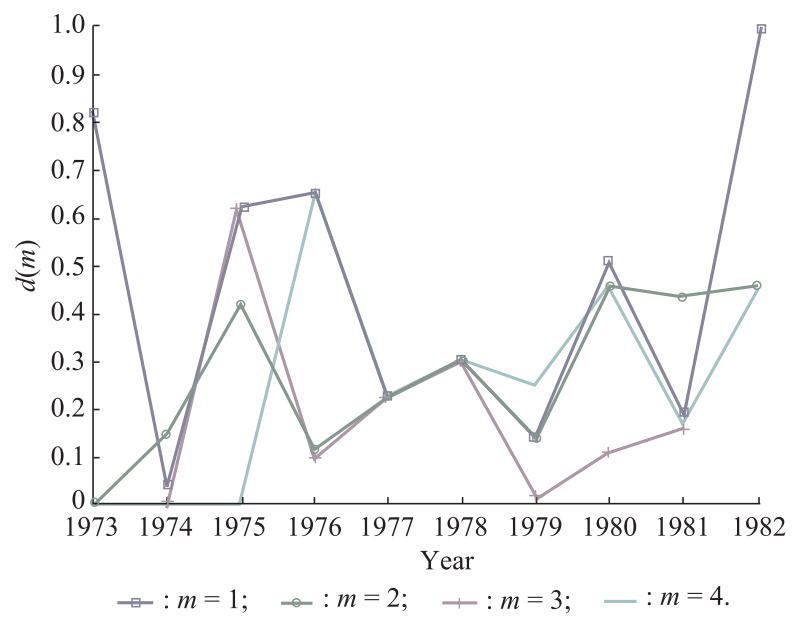

Fig. 5 Intuitionistic fuzzy distance square error

Finally, an appropriate order value $m=3$ based on (11) is obtained and the prediction accuracy is improved by choosing this appropriate order seen from Fig. 5. By the way, there exists a phenomenon that numerous identical $\boldsymbol{R}_{i j}$ appears in the row vectors $\boldsymbol{R}_{I}$ when $m \geqslant 5$ in experiments. We deem that it is an ineffective intuitionistic fuzzy relationship and draw a conclusion that over high order may invalidate $\boldsymbol{R}_{I}$. It is another reason why we need to ascertain an appropriate order.

\subsection{Adaptive partition IFTS forecasting}

Here, an adaptive IFTS forecasting model is stepwise constructed. Because $m=3$ is decided in Section 3.1, the former four data as an example of the 3-order model is illustrated by the forecasting procedure.

(i) The universe of discourse initial partition

Firstly, set $p=6, \Delta A=1000$, and initialize equal partition as follows: $A_{1}=[12500,13$ 500], 
$A_{2}=[13000,14000], A_{3}=[13500,14500], A_{4}=$ [14 000, 15 000], $A_{5}=\left[\begin{array}{lll}14 & 500,15 & 500\end{array}\right]$ and $A_{6}=$ [15 000, 16 000]. Two linguistic fuzzification results are calculated by the interval where the enrollments drop and shown in column 3 of Table 4.

Table 4 Enrollments fuzzification

\begin{tabular}{cccc}
\hline Year & Value & Fuzzification & Membership and non-membership \\
\hline \multirow{2}{*}{1971} & \multirow{2}{*}{13055} & $A_{1}, A_{2}$ & $<0.8842,0.1100>/ u_{1}+$ \\
& & & $<0.0632,0.8900>/ u_{2}$ \\
1972 & 13563 & $A_{2}, A_{3}$ & $<0.8674,0.1260>/ u_{2}+$ \\
& & & $<0.0800,0.8740>/ u_{3}$ \\
1973 & 13867 & $A_{2}, A_{3}$ & $<0.2274,0.7340>/ u_{2}+$ \\
& & & $<0.7200,0.2660>/ u_{3}$ \\
1974 & 14696 & $A_{4}, A_{5}$ & $<0.5874,0.3920>/ u_{4}+$ \\
& & & $<0.3600,0.6080>/ u_{5}$ \\
\hline
\end{tabular}

(ii) Historical time series data intuitionistic fuzzification

Secondly, based on Step 2 of the adaptive partition algorithm and (7), set $\lambda=0.95$, then the former four data of the membership and non-membership functions are calculated and $A_{i}=<0,1>/ u_{j}$ are omitted, as shown in column 4 in Table 4.

(iii) Intuitionistic fuzzy relationship and vector operator matrix calculation

Then, three intuitionistic fuzzy relationships are obtained, namely, $<A_{1}, A_{2} \rightarrow A_{2} A_{3}>,<A_{2} A_{3} \rightarrow$ $A_{2} A_{3}>$ and $<A_{2} A_{3} \rightarrow A_{4} A_{5}>$. According to (15) and (13), $r=1$ and $\boldsymbol{O}(t)=\boldsymbol{D}$ are computed, respectively. The relationship matrix $\boldsymbol{R}_{\mu}$ and $\boldsymbol{R}_{\gamma}$ are calculated by (6).

$$
\boldsymbol{R}_{\mu}=
$$

$\left[\begin{array}{ccccccc}0.8842 & 0.8842 & 0.8842 & 0.8842 & 0.8842 & 0.8842 \\ 0.8674 & 0.8674 & 0.8674 & 0.8674 & 0.8674 & 0.8674 \\ 0.7200 & 0.8674 & 0.7200 & 0.7200 & 0.7200 & 0.7200 \\ 0 & 0.8674 & 0.7200 & 0.5874 & 0.3600 & 0 \\ 0 & 0.8674 & 0.7200 & 0.5874 & 0.3600 & 0 \\ 0 & 0.8674 & 0.7200 & 0.5874 & 0.3600 & 0\end{array}\right]$

$$
\boldsymbol{R}_{\gamma}=
$$

\begin{tabular}{|c|c|c|c|c|c|}
\hline 0.1100 & 0.1100 & 0.1100 & .1100 & 0.1100 & 0.110 \\
\hline 0.1260 & 0.1260 & 0.1260 & 0.1260 & 0.1260 & 0.1260 \\
\hline 0.2660 & 0.1260 & 0.2660 & 0.2660 & 0.2660 & 0.2660 \\
\hline 1 & 0.1260 & 0.2660 & 0.3920 & 0.6080 & 1 \\
\hline 1 & 0.1260 & 0.2660 & 0.3920 & 0.6080 & 1 \\
\hline 1 & 0.1260 & 0.2660 & 0.3920 & 0.6080 & 1 \\
\hline
\end{tabular}

(iv) Forecasting and defuzzification

In succession, $F(t+1)$ is calculated based on (12), forecast fuzzy output $A_{i}, A_{j} \rightarrow A_{i+r}, A_{j+r}$. The fuzzification of next enrollments is $\left\langle A_{5}, A_{6}\right\rangle$. The defuzzification value 15507 is calculated based on (9). Here, choose the membership and non-membership functions of $<A_{5}, A_{6}>$ and $l=2$. (v) Universe of discourse partition updating

The scale of interval is unchanged as the forecasting value is not overflow. The new universe of discourse

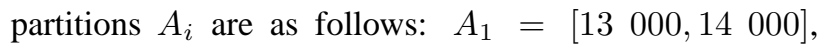
$A_{2}=\left[\begin{array}{lll}13 & 500,14 & 500\end{array}\right], A_{3}=\left[\begin{array}{lll}14 & 000,15 & 000\end{array}\right]$,

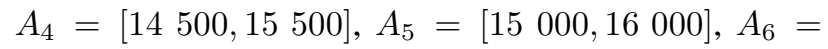
[15 500, 16 500], $A_{7}=$ [16 000, 17 000]. Finally, jump to Step 4 of the adaptive partition algorithm and calculate the rest fuzzification and defuzzification values shown in Table 5, where the numbers in blank denote the update times.

Table 5 Fuzzification forecasting of enrollments

\begin{tabular}{cccc}
\hline Year & Enrollment & Fuzzification & Forecasting \\
\hline 1975 & 15460 & $(\mathbf{0}) A_{5}, A_{6}$ & $(\mathbf{0}) A_{5}, A_{6}$ \\
1976 & 15311 & $(\mathbf{1}) A_{4}, A_{5}$ & $(\mathbf{1}) A_{5}, A_{6}$ \\
1977 & 15603 & $(\mathbf{1}) A_{5}, A_{6}$ & $(\mathbf{1}) A_{5}, A_{6}$ \\
1978 & 15861 & $(\mathbf{1}) A_{5}, A_{6}$ & $(\mathbf{1}) A_{5}, A_{6}$ \\
1979 & 16807 & $(\mathbf{2}) A_{5}, A_{6}$ & $(\mathbf{2}) A_{4}, A_{5}$ \\
1980 & 16919 & $(\mathbf{2}) A_{5}, A_{6}$ & $(\mathbf{2}) A_{6}, A_{7}$ \\
1981 & 16383 & $(\mathbf{2}) A_{4}, A_{5}$ & $(\mathbf{2}) A_{4}, A_{5}$ \\
1982 & 15433 & $(\mathbf{2}) A_{2}, A_{3}$ & $(\mathbf{2}) A_{3}, A_{4}$ \\
1983 & 15497 & $(\mathbf{2}) A_{2}, A_{3}$ & $(\mathbf{2}) A_{2}, A_{3}$ \\
1984 & 15145 & $(\mathbf{2}) A_{2}, A_{3}$ & $(\mathbf{2}) A_{2}, A_{3}$ \\
1985 & 15163 & $(\mathbf{2}) A_{2}, A_{3}$ & $(\mathbf{2}) A_{2}, A_{3}$ \\
1986 & 15984 & $(\mathbf{2}) A_{3}, A_{4}$ & $(\mathbf{2}) A_{2}, A_{3}$ \\
1987 & 16859 & $(\mathbf{2}) A_{5}, A_{6}$ & $(\mathbf{2}) A_{4}, A_{5}$ \\
1988 & 18150 & $(\mathbf{3}) A_{6}, A_{7}$ & $(\mathbf{3}) A_{6}, A_{7}$ \\
1989 & 18970 & $(\mathbf{4}) A_{5}, A_{6}$ & $(\mathbf{4}) A_{4}, A_{5}$ \\
1990 & 19328 & $(\mathbf{4}) A_{6}, A_{7}$ & $(\mathbf{4}) A_{6}, A_{7}$ \\
1991 & 19337 & $(\mathbf{4}) A_{6}, A_{7}$ & $(\mathbf{4}) A_{6}, A_{7}$ \\
1992 & 18876 & $(\mathbf{4}) A_{5}, A_{6}$ & $(\mathbf{4}) A_{6}, A_{7}$ \\
\hline
\end{tabular}

\subsection{Empirical analysis}

Here, we compare forecasting results with those models in $[1,3,5,37,40]$, as shown in Fig. 6. The comparison of square error among different models are shown in Fig. 7 and Fig. 8. These figures show that the model proposed in this paper performs better than those models in $[1,3,40]$. The mean square error (MSE) and average forecasting error rate (AFER) are calculated respectively in Table 6, which indicates the forecasting mean error of our method is lower or almost equal with other methods. It needs to be emphasized that the space complexity may decrease in the adaptive partition model. This experiment will be carried out in next section. Definitions of MSE, the root of mean squared error (RMSE) and AFER are shown as follows, where $f v_{i}$ is the forecasted value, $a v_{i}$ is the actual value, and $f e_{i}$ is the forecasted error.

$$
\mathrm{MSE}=\frac{\sum_{i=1}^{n}\left(f v_{i}-a v_{i}\right)^{2}}{n}
$$




$$
\begin{aligned}
\text { RMSE } & =\sqrt{\frac{\sum_{i=1}^{n}\left(f v_{i}-a v_{i}\right)^{2}}{n}} \\
f e & =\left|\frac{a v-f v}{a v}\right| \\
\text { AFER } & =\frac{\sum f e}{n} \times 100 \% .
\end{aligned}
$$

In the same example, the universe was divided into seven parts [3]. The operation matrix was simple, but the forecasting accuracy was unsatisfied. The model in [36] divided the universe into 14 parts, which improved the forecasting accuracy by increasing space costs. However, the partitions of our model are less than eight and updated four times, which improves complexity and accuracy simultaneously.

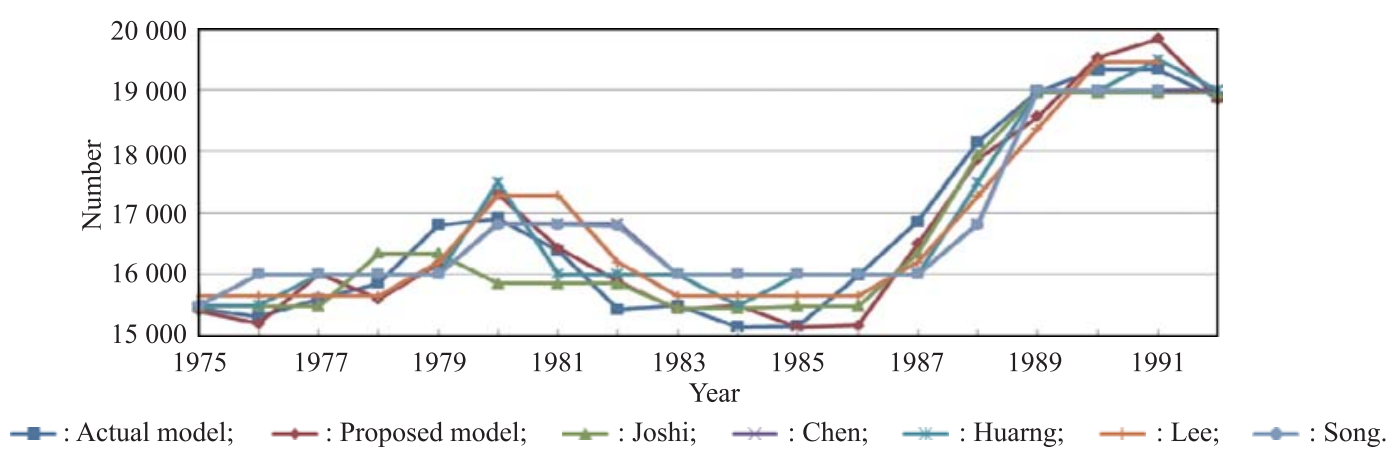

Fig. 6 Forecasting result and comparison

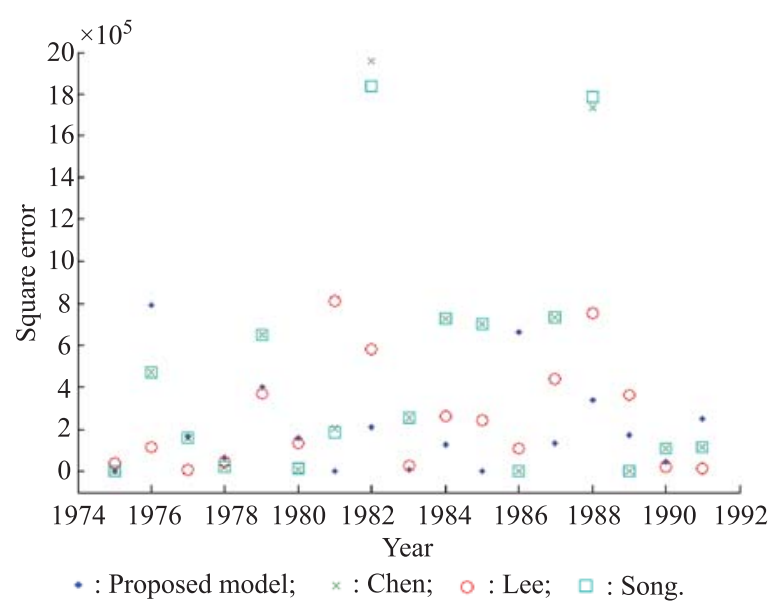

Fig. 7 Comparison of square error 1

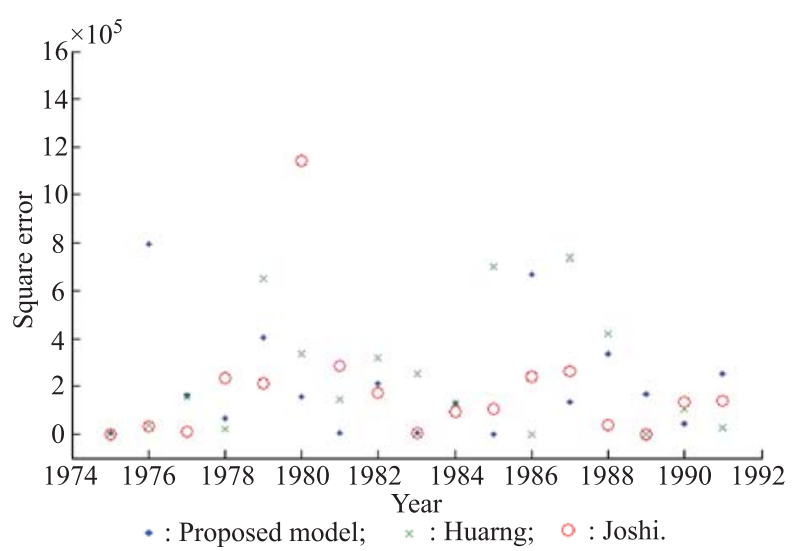

Fig. 8 Comparison of square error 2

Table 6 Comparison of MSE and AFER

\begin{tabular}{ccccccc}
\hline Model & Proposed model & Joshi and Kumar [36] & Chen [3] & Huarng [5] & Lee and Chou [40] & Song and Chissom [1] \\
\hline MSE & 103950 & 175559 & 439421 & 239483 & 240047 & 458438 \\
AFER/\% & 1.58000 & 2.069264 & 2.482540 & 3.21735 & 2.49977 & 3.349670 \\
\hline
\end{tabular}

\section{Forecasting experiments with different patterns}

In former section, the proposed IFTS forecasting model is constructed and applied on enrollments of Alabama University and the empirical analysis shows that our model outperforms other models. Here, we demonstrate the generalization ability of the proposed model by different data patterns.

\subsection{Forecasting seasonal data pattern}

Taiwan Stock Exchange Capitalization Weighted Stock Index (TAIEX) (http://www.twse.com.tw) is a typical seasonal pattern, which is widely used in FTS forecasting $[6,11,19,29,41,42]$. In order to compare with these existing methods, a 4-order adaptive IFTS forecasting model is constructed in training part, where the initial partition is set as $p=8, \Delta A=100$. In above literature, only a 
part of forecasting results are presented, so the comparison results with methods in [6] and [3] from 2000/11/2 to $2000 / 12 / 30$ are shown in columns $1-5$ of Table 7 , and the comparison results with methods in [41] and [19] from $2004 / 11 / 1$ to $2004 / 12 / 31$ are shown in columns $6-10$. As shown in Table 7, the index RMSE of our model is smaller than those methods in [3], [6], [41], and almost equal with [19]. Moreover, we apply the proposed model to forecast the TAIEX from 1990 to 1999 and compare it with methods proposed in $[41,42,29]$. The RMSE of the every year are presented in Table 8 and the smallest value obtained by different models is highlighted in bold. Table 8 shows that 10 years average RMSE of the proposed model is lower than that of $[41,42,29]$. In conclusion, the proposed IFTS model outperforms methods above in such seasonal pattern.

Table 7 Parts of TAIEX forecasting result

\begin{tabular}{|c|c|c|c|c|c|c|c|c|c|}
\hline Date & Actual model & Hybrid [6] & Chen [3] & Proposed model & Date & Actual model & Chen [41] & Chen [19] & Proposed model \\
\hline $2000 / 11 / 2$ & 5626 & 5550 & 5300 & 5524.34 & $2004 / 11 / 1$ & 5656.17 & 5718.09 & - & - \\
\hline $2000 / 11 / 3$ & 5796 & 5650 & 5750 & 5742.54 & $2004 / 11 / 2$ & 5759.61 & 5658.97 & - & - \\
\hline $2000 / 11 / 4$ & 5677 & 5750 & 5450 & 5514.26 & $2004 / 11 / 3$ & 5862.85 & 5754.24 & 5756.8 & - \\
\hline $2000 / 11 / 6$ & 5657 & 5650 & 5750 & 5658.21 & $2004 / 11 / 4$ & 5860.73 & 5863.09 & 5865.05 & - \\
\hline $2000 / 11 / 7$ & 5877 & 5650 & 5750 & 5865.27 & $2004 / 11 / 5$ & 5931.31 & 5868.04 & 5868.44 & 5897.32 \\
\hline $2000 / 11 / 8$ & 6067 & 5850 & 5750 & 5924.15 & $2004 / 11 / 8$ & 5937.46 & 5927.67 & 5935.51 & 5932.14 \\
\hline $2000 / 11 / 10$ & 6088 & 5950 & 6075 & 6035.24 & $2004 / 11 / 10$ & 5948.49 & 5942.85 & 5940.7 & 5948.36 \\
\hline $2000 / 11 / 13$ & 5793 & 5950 & 6075 & 5835.12 & $2004 / 11 / 11$ & 5874.52 & 5952.69 & 5945.72 & 5936.32 \\
\hline $2000 / 11 / 14$ & 5772 & 5750 & 5450 & 5678.65 & $2004 / 11 / 12$ & 5917.16 & 5882.56 & 5873.1 & 5914.05 \\
\hline $2000 / 11 / 15$ & 5737 & 5750 & 5450 & 5724.04 & $2004 / 11 / 15$ & 5906.69 & 5931.63 & 5919.36 & 5901.52 \\
\hline $2000 / 11 / 16$ & 5454 & 5750 & 5450 & 5514.83 & $2004 / 11 / 16$ & 5910.85 & 5908.03 & 5905.27 & 5896.91 \\
\hline $2000 / 11 / 17$ & 5351 & 5550 & 5300 & 5451.29 & $2004 / 11 / 17$ & 6028.68 & 5912.13 & 5905.27 & 5943.21 \\
\hline $2000 / 11 / 20$ & 4845 & 5150 & 5150 & 4836.21 & 2004/11/19 & 6026.55 & 6042.75 & 6051.69 & 6054.36 \\
\hline $2000 / 11 / 21$ & 5103 & 4850 & 4850 & 5178.36 & $2004 / 11 / 22$ & 5838.42 & 6010.72 & 6051.69 & 5863.14 \\
\hline $2000 / 11 / 22$ & 5130 & 5150 & 5150 & 5145.07 & $2004 / 11 / 23$ & 5851.1 & 5862.23 & 5837 & 5774.63 \\
\hline $2000 / 11 / 23$ & 5146 & 5150 & 5150 & 5136.89 & $2004 / 11 / 24$ & 5911.31 & 5855.49 & 5853.3 & 5712.24 \\
\hline $2000 / 11 / 24$ & 5419 & 5150 & 5150 & 5336.15 & $2004 / 11 / 25$ & 5855.24 & 5911.03 & 5913.51 & 5741.26 \\
\hline $2000 / 11 / 27$ & 5433 & 5550 & 5300 & 5324.84 & $2004 / 11 / 26$ & 5778.26 & 5858.01 & 5862.16 & 5873.24 \\
\hline $2000 / 11 / 28$ & 5362 & 5550 & 5300 & 5382.80 & $2004 / 11 / 29$ & 5785.26 & 5773.75 & 5785.57 & 5843.84 \\
\hline $2000 / 11 / 29$ & 5319 & 5350 & 5350 & 5314.18 & $2004 / 11 / 30$ & 5844.76 & 5783.93 & 5780.76 & 5815.39 \\
\hline $2000 / 11 / 30$ & 5256 & 5350 & 5250 & 5221.92 & $2004 / 12 / 1$ & 5798.62 & 5844.68 & 5841.95 & 5658.85 \\
\hline $2000 / 12 / 1$ & 5342 & 5250 & 5350 & 5336.24 & $2004 / 12 / 2$ & 5867.95 & 5817.26 & 5797.2 & 5748.36 \\
\hline $2000 / 12 / 2$ & 5811 & 5350 & 5250 & 5563.36 & $2004 / 12 / 3$ & 5893.27 & 5861.34 & 5865.14 & 5896.14 \\
\hline $2000 / 12 / 4$ & 5174 & 5250 & 5250 & 5114.66 & $2004 / 12 / 6$ & 5919.17 & 5897.53 & 5895.47 & 5954.36 \\
\hline $2000 / 12 / 5$ & 5199 & 5150 & 5150 & 5214.47 & $2004 / 12 / 7$ & 5925.28 & 5916.83 & 5916.36 & 5963.17 \\
\hline $2000 / 12 / 6$ & 5170 & 5150 & 5150 & 5363.31 & $2004 / 12 / 8$ & 5892.51 & 5910.24 & 5920.78 & 5847.54 \\
\hline $2000 / 12 / 11$ & 5284 & 5250 & 5250 & 5363.91 & $2004 / 12 / 13$ & 5878.89 & 5915.62 & 5910.71 & 5912.4 \\
\hline $2000 / 12 / 12$ & 5380 & 5250 & 5250 & 5236.31 & $2004 / 12 / 14$ & 5909.65 & 5882.3 & 5877.47 & 5914.04 \\
\hline $2000 / 12 / 13$ & 5384 & 5350 & 5350 & 5342.78 & $2004 / 12 / 15$ & 6002.58 & 5911.86 & 5911.85 & 6082.16 \\
\hline $2000 / 12 / 14$ & 5320 & 5350 & 5350 & 5341.03 & $2004 / 12 / 16$ & 6019.23 & 6002.5 & 6004.78 & 6024.15 \\
\hline $2000 / 12 / 15$ & 5224 & 5350 & 5350 & 5324.0 & $2004 / 12 / 17$ & 6009.32 & 5027.15 & 6026.94 & 6254.36 \\
\hline $2000 / 12 / 16$ & 5134 & 5250 & 5250 & 5436.54 & $2004 / 12 / 20$ & 5985.94 & 6012.86 & 6004.82 & 6014.25 \\
\hline $2000 / 12 / 18$ & 5055 & 5150 & 5150 & 5234.63 & $2004 / 12 / 21$ & 5987.85 & 5999.1 & 5984.52 & 6114.91 \\
\hline $2000 / 12 / 19$ & 5040 & 5350 & 5450 & 5123.14 & $2004 / 12 / 22$ & 6001.52 & 6005.92 & 5995.56 & 6011.38 \\
\hline $2000 / 12 / 20$ & 4947 & 5350 & 5450 & 4721.39 & $2004 / 12 / 23$ & 5997.67 & 6005.86 & 6009.23 & 5954.1 \\
\hline $2000 / 12 / 21$ & 4817 & 4950 & 4950 & 4745.86 & $2004 / 12 / 24$ & 6019.42 & 5997.68 & 6005.38 & 5987.29 \\
\hline $2000 / 12 / 22$ & 4811 & 4850 & 4850 & 4984.25 & $2004 / 12 / 27$ & 5985.94 & 6026.35 & 6016.65 & 6087.76 \\
\hline $2000 / 12 / 26$ & 4721 & 4850 & 4850 & 4832.12 & $2004 / 12 / 28$ & 6000.57 & 5987.87 & 5985.02 & 6010.47 \\
\hline $2000 / 12 / 27$ & 4614 & 4750 & 4750 & 4654.32 & $2004 / 12 / 29$ & 6088.49 & 6018.68 & 6008.28 & 6000.14 \\
\hline $2000 / 12 / 28$ & 4974 & 4650 & 4650 & 4924.24 & $2004 / 12 / 30$ & 6100.86 & 6086.83 & 6085.68 & 6004.2 \\
\hline $2000 / 12 / 29$ & 4743 & 4750 & 4750 & 4785.36 & $2004 / 12 / 31$ & 6139.69 & 6099.45 & 6098.05 & 6124.85 \\
\hline $2000 / 12 / 30$ & 4739 & 4750 & 4750 & 4612.01 & - & - & - & - & - \\
\hline RMSE & - & 173.46 & 200.32 & 127.88 & RMSE & - & 155.65 & 53.3617 & 77.57 \\
\hline
\end{tabular}


Table 8 Comparison of RMSE for different model

\begin{tabular}{|c|c|c|c|c|c|c|c|c|c|c|c|}
\hline Model & 1990 & 1991 & 1992 & 1993 & 1994 & 1995 & 1996 & 1997 & 1998 & 1999 & Average \\
\hline Chen [41] & 174.62 & 43.22 & 42.66 & 104.17 & 94.6 & 54.24 & 50.5 & 138.51 & 117.87 & 101.33 & 92.17 \\
\hline Chen and Kao [42] & 156.47 & 56.50 & 36.45 & 126.45 & 62.57 & 105.52 & 51.50 & 125.33 & 104.12 & 87.63 & 91.25 \\
\hline Cai and Zhang [29] & 187.10 & 39.58 & 39.37 & 101.80 & 76.32 & 56.05 & 49.45 & 123.98 & 118.41 & 102.34 & 89.44 \\
\hline Proposed model & 148.17 & 40.14 & 38.14 & 98.47 & 84.69 & 55.78 & 51.96 & 89.87 & 122.87 & 76.02 & 80.61 \\
\hline
\end{tabular}

\subsection{Forecasting secular trend data pattern}

To show its wide applicability, our method is implemented on another pattern, secular trend model. Monthly total retail sale of social consumer goods (TRSSCG) is a typical model which is obtained from National Bureau of Statistics of China (www.data.stats.gov.cn) in this section. The specimens include 96 data from January 1994 to December 2011. The parts of data are shown in Table 9.

Table 9 Parts data of TRSSCG

billion

\begin{tabular}{|c|c|c|c|c|c|c|c|c|}
\hline Date & Value & Date & Value & Date & Value & $\ldots$ & Date & Value \\
\hline Jan 1994 & 120.85 & Jan 1995 & 160.83 & Jan 1996 & 192.45 & $\cdots$ & Jan 2011 & 1524.90 \\
\hline Feb 1994 & 117.81 & Feb 1995 & 150.53 & Feb 1996 & 192.67 & & Feb 2011 & 1376.91 \\
\hline Mar 1994 & 118.30 & Mar 1995 & 154.65 & Mar 1996 & 187.52 & & Mar 2011 & 1358.80 \\
\hline Apr 1994 & 118.59 & Apr 1995 & 154.65 & Apr 1996 & 186.98 & & Apr 2011 & 1364.90 \\
\hline May 1994 & 122.98 & May 1995 & 158.77 & May 1996 & 191.37 & & May 2011 & 1469.68 \\
\hline Jun 1994 & 129.81 & Jun 1995 & 164.96 & Jun 1996 & 198.19 & $\ldots$ & Jun2011 & 1456.51 \\
\hline Jul 1994 & 126.81 & Jul 1995 & 162.90 & Jul 1996 & 190.40 & & Jul 2011 & 1440.80 \\
\hline Aug 1994 & 130.31 & Aug 1995 & 164.96 & Aug 1996 & 193.19 & & Aug 2011 & 1470.50 \\
\hline Sept 1994 & 141.47 & Sept 1995 & 177.33 & Sep 1996 & 210.04 & & Sep 2011 & 1586.51 \\
\hline Oct 1994 & 146.32 & Oct 1995 & 181.46 & Oct 1996 & 216.57 & & Oct 2011 & 1654.64 \\
\hline Nov 1994 & 157.44 & Nov 1995 & 193.83 & Nov 1996 & 230.86 & & Nov 2011 & 1612.89 \\
\hline Dec 1994 & 195.78 & Dec 1995 & 237.13 & Dec 1996 & 287.17 & $\ldots$ & Dec 2011 & 1773.97 \\
\hline
\end{tabular}

Firstly, set the initial partition as $p=6, \Delta A=20$, $A_{1}=[100,120], A_{2}=[110,130], A_{3}=[120,140]$, $A_{4}=[130,150], A_{5}=[140,160]$ and $A_{6}=[150,170]$. In the forecasting process, the value of interval updates three times, i.e., $\Delta A_{1}=2 \times 20=40, \Delta A_{2}=2^{2} \times 20=80$, $\Delta A_{3}=2^{3} \times 20=160$. The comparison between actual values and forecast results is shown in Fig. 9, which indicates that the adaptive partition IFTS forecasting model performs excellently on prediction of TRSSCG. The comparisons of square error with other methods are shown in Fig. 10 and Fig. 11. In other methods, set the scale of partition as 10, and the square error distribution figure illustrates that the proposed model makes lower error.

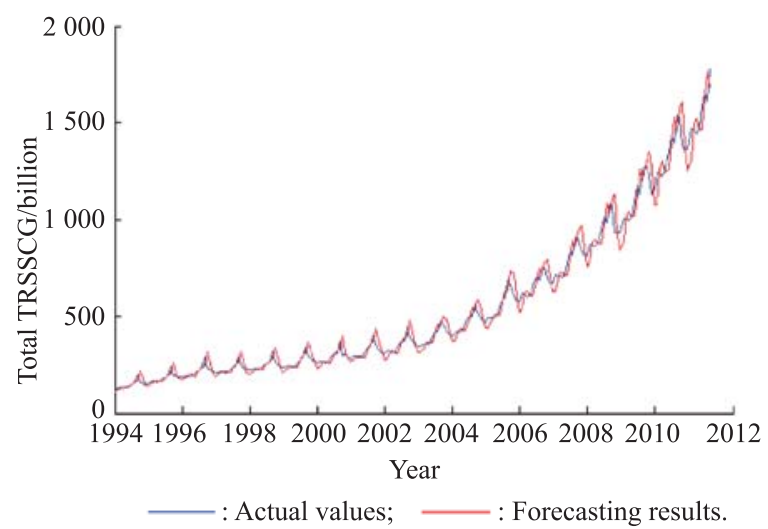

Fig. 9 Forecast result of TRSSCG

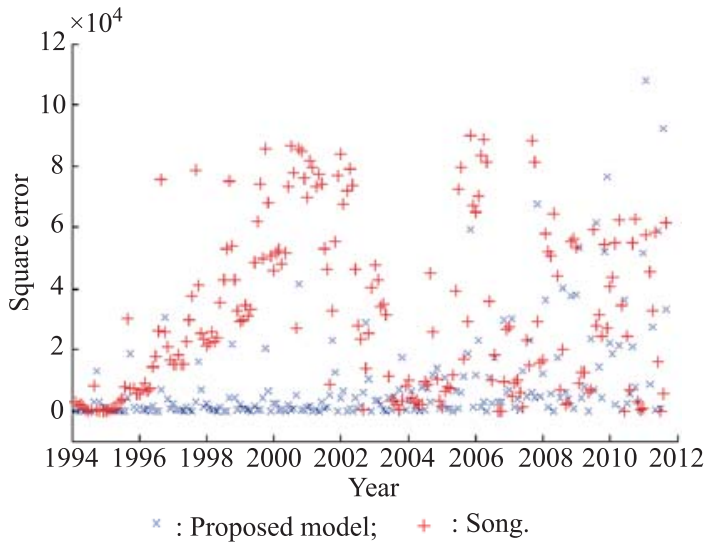

Fig. 10 Comparison of square error on TRSSCG (1)

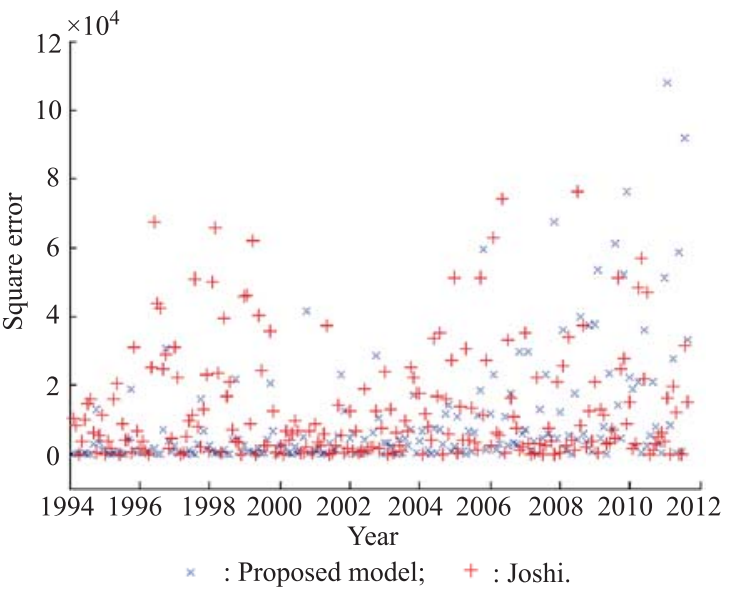

Fig. 11 Comparison of square error on TRSSCG (2) 
Considering the fact that AFER is influenced by the scale of date sets, we compare the proposed model with others with index MSE only, as shown in Table 10. The index MSE of our model is the minimum among four methods.

Table 10 Comparison of MSE in TRSSCG

\begin{tabular}{ccccc}
\hline Model & $\begin{array}{c}\text { Proposed } \\
\text { model }\end{array}$ & $\begin{array}{c}\text { Song and } \\
\text { Chissom [1] }\end{array}$ & $\begin{array}{c}\text { Lee and } \\
\text { Chou [40] }\end{array}$ & $\begin{array}{c}\text { Joshi and } \\
\text { Kumar [36] }\end{array}$ \\
\hline MSE & 7308 & 32452 & 22603 & 11747 \\
\hline
\end{tabular}

\subsection{Empirical analysis}

In order to evaluate the computational complexity of different models, we present the time complexity of each method in Table 11. Song's, Lee's and Joshi's models divide every time series into $n$ parts. Chen's, Huarng's and Gangwar's models rely on partition of universe of discourse $n$ and the fuzzy logical relationships groups $g$, and each FLT needs to be divided. The proposed model needs to calculate the optical order number $m$, whose computational complexity is equal with other models'.

Based on the particular experiments analyzed above, we can see that the performance of forecasting models presented in $[1,36,40]$ is not bad when applied on the student enrollments data. However, the forecasting MSE is high when applied to the TRSSCG data. Our model obtains effective forecasting results on enrollments dataset and performs better than $[3,6,41]$ on seasonal dataset (e.g. TAIEX). The experiments show that the performance of the same methods may be different when the forecasting objects change. The traditional methods are suitable for smooth pattern only. It is proved that the proposed adaptive IFTS model is appropriate for different situations by adjusting partitions and intervals of universe.

Table 11 Comparison of computational complexity

\begin{tabular}{cc}
\hline Model & $T(n)$ \\
\hline Song's model [1] & $O\left(n^{2}\right)$ \\
Chen's model [3] & $O\left(g n^{2}\right)$ \\
Huarng's model [5] & $O\left(g n^{2}\right)$ \\
Gangwar's model [16] & $O\left(g n^{2}\right)$ \\
Joshi's [36] & $O\left(n^{2}\right)$ \\
Lee's [40] & $O\left(n^{2}\right)$ \\
Proposed model & $O\left(m+n^{2}\right)$ \\
\hline
\end{tabular}

\section{Conclusions}

In this paper, we construct an IFTS forecasting model with order deciding and adaptive partition algorithms. The proposed model can adjust partitions and intervals adaptively by forecasting the vector operator matrix that is validated effectively on enrollments of Alabama. We implement the proposed model on two different dataset patterns.
The TAIEX experimental results show that our model performs better than other relevant methods. The other pattern is the secular trend dataset. We experiment our model on almost eight years' monthly TRSSCG, and both the forecasting accuracy and MSE are the most optimized one. The experimental results illustrate the wide applicability and generality of the proposed model in different patterns.

\section{References}

[1] Q. Song, B. S. Chissom. Forecasting enrollments with fuzzy time series - Part I. Fuzzy Sets Systems, 1993, 54(1): 1 - 9.

[2] Q. Song, B. S. Chissom. Forecasting enrollments with fuzzy time series - Part II. Fuzzy Sets Systems, 1994, 62(1): 1-8.

[3] S. M. Chen. Forecasting enrollments based on fuzzy time series. Fuzzy Sets Systems, 1996, 81(3): 311-319.

[4] J. R. Hwang, S. M. Chen, C. H. Lee. Handling forecasting problems using fuzzy time series. Fuzzy Sets Systems, 1998, 100(2): $217-228$.

[5] K. Huarng. Heuristic models of f uzzy time series for forecasting. Fuzzy Sets Systems, 2001, 123(2): 369-386.

[6] K. Huarng, H. K. Yu. The application of neural networks to forecast fuzzy time series. Physica A, 2006, 363(2): $481-491$.

[7] J. Park, D. J. Lee, C. K. Song, et al. TAIFEX and KOSPI 200 forecasting based on two-factor high-order fuzzy time series and particle swarm optimization. Expert Systems with Applications, 2010, 37(2): 959-967.

[8] Q. Song. A note on fuzzy time series model relation with sample autocorrelation functions. Cybernetics and Systems, 2003, 34(2): $93-107$.

[9] C. H. Cheng, J. R. Chang. Entropy-based and trapezoid fuzzification based fuzzy time series approaches for forecasting IT project cost. Technological Forecasting and Social Change, 2006, 73(5): 524-542.

[10] T. A. Jilani, S. M. A. Burney. Multivariate stochastic fuzzy forecasting models. Expert Systems with Applications, 2008, 35(3): $691-700$.

[11] J. W. Liu, T. L. Chen, C. H. Cheng, et al. Adaptive-expectation based multi-attribute FTS model for forecasting TAIEX. Computers and Mathematics with Applications, 2010, 59(2): 195 802.

[12] S. M. Chen. Forecasting enrollments based on high-order fuzzy time series. Cybernetics and System, 2002, 33(1): 1-8.

[13] C. M. Own, P. T. Yu. Forecasting fuzzy time series on a heuristic high-order model. Cybernetics and Systems, 2005, 36(7): $705-717$.

[14] L. W. Lee, L. H. Wang, S. M. Chen. Handling forecasting problems based on two-factors high-order fuzzy time series. IEEE Trans. on Fuzzy Systems, 2006, 14(3): 468 - 477.

[15] P. Singh, B. Borah. High-order fuzzy-neuro expert system for time series forecasting. Knowledge-Based Systems, 2013, 46(1): $12-21$.

[16] S. S. Gangwar, S. Kumar. Partitions based computational method for high-order fuzzy time series forecasting. Expert Systems with Applications, 2012, 39(15): 12158 - 12164.

[17] S. M. Chen, K. Tanuwijaya. Multivariate fuzzy forecasting based on fuzzy time series and automatic clustering techniques. Expert Systems with Applications, 2011, 38(8): $10594-10605$.

[18] S. Askari, N. Montazerin. A high-order multi-variable fuzzy time series forecasting algorithm based on fuzzy clustering. Expert Systems with Applications, 2015, 42(9): 2121 - 2135.

[19] S. M. Chen, S. H. Chen. Fuzzy forecasting based on two- 
factors second-order fuzzy-trend logical relationship groups and the probabilities of trends of fuzzy logical relationships. IEEE Trans. on Cybernetics, 2015, 45(3): 405-417.

[20] C. H. Cheng, G. W. Cheng, J. W. Wang. Multi-attribute fuzzy time series method based on fuzzy clustering. Expert Systems with Applications, 2008, 34(2): 1235-1242.

[21] S. T. Li, Y. C. Cheng, S. Y. Lin. An FCM-based deterministic forecasting model for fuzzy time series. Computers and Mathematics with Applications, 2008, 56(12): 3052-3063.

[22] E. Egrioglu, C. H. Aladag, U. Yolcu. Fuzzy time series forecasting with a novel hybrid approach combining fuzzy cmeans and neural networks. Expert Systems with Applications, 2013, 40(3): $854-857$.

[23] H. Izakian, W. Pedrycz, I. Jamal. Fuzzy clustering of time series data using dynamic time warping distance. Engineering Applications of Artificial Intelligence, 2015, 39(1): 235-244.

[24] Y. L. Huang, S. J. Horng, M. X. He, et al. A hybrid forecasting model for enrollments based on aggregated fuzzy time series and particle swarm optimization. Expert Systems with Applications, 2011, 38(7): 8014-8023.

[25] I. H. Kuo, S. J. Horng, T. W. Kao, et al. An improved method for forecasting enrollments based on fuzzy time series and particle swarm optimization. Expert Systems with Application, 2009, 36(3): 6108-6117.

[26] M. Avazbeigi, S. H. Doulabi, B. Karimi. Choosing the appropriate order in fuzzy time series: a new $N$-factor fuzzy time series for prediction of the auto industry production. Expert Systems with Applications, 2010, 37(8): 5630-5639.

[27] L. Z. Wang, X. D. Liu, W. Pedrycz. Effective intervals determined by information granules to improve forecasting in fuzzy time series. Expert Systems with Applications, 2013, 40(14): $1465-1470$.

[28] L. Wei, X. Y. Chen, W. Pedrycz, et al. Using interval information granules to improve forecasting in fuzzy time series. International Journal of Approximate Reasoning, 2015, 57(11): $1-18$.

[29] Q. S. Cai, D. F. Zhang, W. Zhang. A new fuzzy time series forecasting model combined with ant colony optimization and auto-regression. Knowledge-Based Systems, 2015, 74(11): 1 68.

[30] R. C. Tsaur, J. C. Yang, H. F. Wang. Fuzzy relation analysis in fuzzy time series model. Computers and Mathematics with Applications, 2005, 49(2): 539-548.

[31] H. T. Liu, M. L. Wei. An improved fuzzy forecasting method for seasonal time series. Expert Systems with Applications, 2010, 37(2): 6310-6318.

[32] S. T. Li, Y. C. Cheng, S. Y. Lin. A FCM-based deterministic forecasting model for fuzzy time series. Computers and Mathematics with Applications, 2008, 56(12): 3052 - 3063.

[33] Y. K. Bang, C. H. Lee. Fuzzy time series prediction using hierarchical clustering algorithms. Expert Systems with Application, 2011, 38(4): 4312-4325.

[34] K. Atanassov. Intutionistic fuzzy sets. Fuzzy Sets and Systems, 1986, 20(1): 87-96.

[35] O. Castillo, A. Alanis, M. Garcia, et al. An intuitionistic fuzzy system for time series analysis in plant monitoring and diagnosis. Applied Soft Computing, 2007, 7(4): 1227-1233.

[36] B. P. Joshi, S. Kumar. Intuitionistic fuzzy sets based method for fuzzy time series forecasting. Cybernetics and Systems, 2012, 43(1): 34-47.

[37] S. S. Gangwar, S. Kumar. Probabilistic and intuitionistic fuzzy sets-based method for fuzzy time series forecasting. Cybernetics and Systems, 2014, 45(4): 349-361.

[38] K. Q. Zheng, Y. J. Lei, R. Wang, et al. Modeling and application of IFTS. Control and Decision, 2013, 28(10): $1525-$ 1530.

[39] K. Q. Zheng, Y. J. Lei, R. Wang, et al. Method of long-term IFTS forecasting based on parameter adaptation. Systems Engineering and Electronics, 2014, 36(1): 99-104. (in Chinese)

[40] H. S. Lee, M. T. Chou. Fuzzy forecasting based on fuzzy time series. International Journal of Computer Mathematics, 2004, 81(7): $781-789$

[41] S. M. Chen, H. P. Chu. TAIEX forecasting using fuzzy time series and automatically generated weights of multiple factors. IEEE Trans. on Systems, Man, and Cybernetics-Part A: Systems and Humans, 2012, 42(6): 1485 - 1495.

[42] S. M. Chen, P. Y. Kao. TAIEX forecasting based on fuzzy time series, particle swarm optimization techniques and support vector machines. Information Sciences, 2013, 247(1): 62-71.

\section{Biographies}

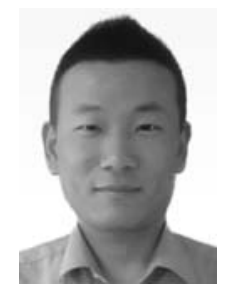

Xiaoshi Fan was born in 1988. He is a doctoral candidate in Air and Missile Defense College, Air Force Engineering University. He is with Department of Computer Science and Technology. His current research interests are fuzzy information systems, intelligence information processing and network information security.

E-mail: fan_xs@126.com

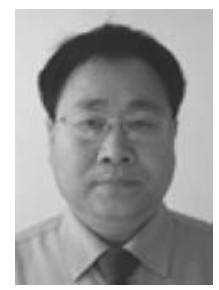

Yingjie Lei was born in 1956. He is a professor in Air and Missile Defense College, Air Force Engineering University. His research interests are network information security and intelligence information processing.

E-mail: leiyjie@163.com

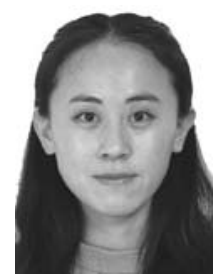

Yanan Wang was born in 1988. She is a doctor in Air and Missile Defense College, Air Force Engineering University. Her research interests are network information security and intelligence information processing.

E-mail: wyn1988814@163.com 